\title{
Bulk and shear viscosities for the Gribov-Zwanziger plasma
}

\author{
Wojciech Florkowski ${ }^{1, a}$ \\ ${ }^{1}$ Institute of Nuclear Physics, Polish Academy of Sciences \\ ul. Radzikowskiego 152, 31-342 Kraków, Poland
}

\begin{abstract}
The concept of the Gribov-Zwanziger plasma is introduced and used to calculate the bulk and shear viscosities of the system of gluons. The kinetic coefficients are obtained in two different ways which are shown to yield equivalent results.
\end{abstract}

\section{Introduction}

In this contribution I report on the recent results obtained in collaboration with Radoslaw Ryblewski, Nan Su, and Konrad Tywoniuk [1,2] describing the form of the bulk and shear viscosity coefficients for the Gribov-Zwanziger (GZ) plasma. The concept of the GZ plasma follows from the use of the dispersion relation

$$
E(\boldsymbol{k})=\sqrt{\boldsymbol{k}^{2}+\frac{\gamma_{\mathrm{G}}^{4}}{\boldsymbol{k}^{2}}}
$$

for the gluons [3]. Here $\boldsymbol{k}$ is the three-momentum of gluons and $E$ denotes their energy. The parameter $\gamma_{\mathrm{G}}$ appears during the quantisation of the Yang-Mills theory. Equation (1) has been derived for the first time by Gribov in his famous paper [3]. It takes into account the expected behavior of gluons in the infrared, namely, a large energy cost connected with the excitation of soft modes and the corresponding reduction of the physical phase space [3-7]. The use of (1) suggests that interacting massless gluons are equivalent to non-interacting gluons having this very special form of the dispersion relation.

Thermodynamic properties of the system with the particles obeying Eq. (1) have been worked out in Refs. [8, 9]. The main results follow directly from the use of (1) in the Bose-Einstein distribution function

$$
f_{\mathrm{GZ}}=\frac{1}{\exp (E(\boldsymbol{k}) / T)-1},
$$

where $T$ is the system's temperature. This approach provides good agreement with the lattice results on thermodynamic variables [10]. Encouraged by the success connected with the use of the dispersion relation (1) in equilibrium, in Refs. [1,2] we have made an attempt to construct a simple kinetic theory that incorporates (1) and may describe processes out of equilibrium. In particular, in [1] we have concentrated on the calculation of the bulk viscosity which attracts more and more attention in the last years [11-15].

\footnotetext{
ae-mail: wojciech.florkowski@ifj.edu.pl
} 


\section{Lorentz covariance and boost-invariance}

\subsection{Reimplementation of Lorentz covariance}

At first it should be emphasised that the original dispersion relation (1) is derived in the Coulomb gauge which explicitly breaks Lorentz invariance. In order to regain a covariant framework, that is required for description of relativistic fluids, one has to make certain assumptions about the Lorentz transformation properties of the quantities that appear in Eq. (1). The strategy to recover the covariant description is not straightforward and we discuss this problem in greater detail in Ref. [2].

We choose the procedure where the Gribov dispersion relation (1) is transformed into the following covariant expression $[1,2]$

$$
E(k \cdot u)=\sqrt{(k \cdot u)^{2}+\frac{\gamma_{\mathrm{G}}^{4}}{(k \cdot u)^{2}}} .
$$

Here $u$ is the four-velocity of the fluid element. We assume that the Coulomb gauge as well as the in-medium value of the Gribov parameter $\gamma_{\mathrm{G}}$ are fixed in the local rest frame where $u^{\mu}=(1,0,0,0)$. We introduce $k^{0} \equiv|\boldsymbol{k}|$, which is the magnitude of the three-vector $\boldsymbol{k} \equiv\left(k_{x}, k_{y}, k_{\|}\right)$, and $k_{\perp}=\sqrt{k_{x}^{2}+k_{y}^{2}}$ such that the resulting four-vector $k^{\mu}=\left(k^{0}, \boldsymbol{k}\right)$ has standard Lorentz transformation properties with $k^{2}=k \cdot k=0$.

\subsection{Boost-invariance}

One of the simplest ways to obtain the formulas for the kinetic coefficients is to analyse a $(0+1)$ dimensional, boost-invariant and transversally homogeneous system. We stress that in contrast to the problem of Lorentz covariance, which is the fundamental issue connected with the use of the Coulomb gauge, imposing boost-invariance is just a technical method to facilitate our manipulations.

The boost-invariant systems are characterised by the flow vector $u^{\mu}=(t / \tau, 0,0, z / \tau)$ [16]. In this case, all scalar functions of space and time depend only on the longitudinal proper time $\tau=\sqrt{t^{2}-z^{2}}$. One may furthermore introduce the boost-invariant variables $v=k^{0} t-k_{\|} z$ and $w=k_{\|} t-k^{0} z$ [17]. In this case the energy of the particle is given by the formula

$$
E\left(\tau, w, k_{\perp}\right)=\sqrt{\frac{w^{2}}{\tau^{2}}+k_{\perp}^{2}+\frac{\gamma_{\mathrm{G}}^{4}}{\frac{w^{2}}{\tau^{2}}+k_{\perp}^{2}}} .
$$

The phase space integration measure in the three-momentum space can be written correspondingly as

$$
\int \mathrm{d} K(\ldots)=\frac{g_{0}}{(2 \pi)^{3}} \int_{-\infty}^{\infty} \frac{\mathrm{d} w}{\tau} \int \mathrm{d}^{2} k_{\perp}(\ldots),
$$

where $g_{0}$ is the number of internal degrees of freedom. We note that the phase space distribution function $f$, which is also a Lorentz scalar, may depend in our case only on $\tau, w$ and $k_{\perp}$, namely $f=f\left(\tau, w, k_{\perp}\right)[17]$.

\section{Kinetic equation}

\subsection{Relaxation time approximation}

The arguments presented in $[1,2]$ suggest that we can use the standard kinetic equation in the relaxation-time approximation (RTA) of the form [18-20]

$$
\frac{\partial f\left(\tau, w, k_{\perp}\right)}{\partial \tau}=\frac{f_{\mathrm{GZ}}\left(\tau, w, k_{\perp}\right)-f\left(\tau, w, k_{\perp}\right)}{\tau_{\mathrm{rel}}(\tau)},
$$


where $\tau_{\text {rel }}$ is the relaxation time and the (effective) temperature $T$ appearing in the equilibrium distribution function $f_{\mathrm{GZ}}$ is determined from the equation

$$
\int \mathrm{d} K E\left(\tau, w, k_{\perp}\right) f_{\mathrm{GZ}}\left(\tau, w, k_{\perp}\right)=\int \mathrm{d} K E\left(\tau, w, k_{\perp}\right) f\left(\tau, w, k_{\perp}\right) .
$$

Equation (7) is known as the Landau matching condition for the energy. The formal solution of Eq. (6) is [21-23]

$$
f\left(\tau, w, k_{\perp}\right)=f_{0}\left(w, k_{\perp}\right) D\left(\tau, \tau_{0}\right)+\int_{\tau_{0}}^{\tau} \frac{\mathrm{d} \tau^{\prime}}{\tau_{\mathrm{rel}}\left(\tau^{\prime}\right)} D\left(\tau, \tau^{\prime}\right) f_{\mathrm{GZ}}\left(\tau^{\prime}, w, k_{\perp}\right),
$$

where the damping function $D\left(\tau_{2}, \tau_{1}\right)$ has the form

$$
D\left(\tau_{2}, \tau_{1}\right)=\exp \left[-\int_{\tau_{1}}^{\tau_{2}} \frac{\mathrm{d} \tau}{\tau_{\text {rel }}(\tau)}\right]
$$

Inserting the solution (8) into the Landau matching condition (7) allows us to find the time dependence of the system's temperature, $T(\tau)$. This, in turn, when used back in (8), allows us to find the time dependence of various system's characteristics such as the energy density and transverse and longitudinal pressures.

\subsection{Linear response method}

Besides the exact treatment of the kinetic equation that has been sketched above, we may use the linear respond method. In this case we seek the solution of Eq. (6) in the form

$$
f \approx f_{\mathrm{GZ}}+\delta f+\cdots,
$$

where $\delta f=-\tau_{\text {rel }} \mathrm{d} f_{\mathrm{Gz}} / \mathrm{d} \tau$. This leads directly to the following result

$$
\delta f=-\frac{E \tau_{\mathrm{rel}}}{T \tau}\left\{\frac{w^{2}}{E^{2} \tau^{2}}\left[1-\frac{\gamma_{\mathrm{G}}^{4}}{\left(\frac{w^{2}}{\tau^{2}}+k_{\perp}^{2}\right)^{2}}\right]+c_{s}^{2}\right\} f_{\mathrm{GZ}}\left(1+f_{\mathrm{GZ}}\right) .
$$

The speed of sound squared, $c_{s}^{2}$, that appears in (11) can be obtained from the equation of state. The correction to the distribution function (11) can be used further to obtain the shear tensor $\pi^{\mu \nu}$ and the bulk pressure $\Pi$. These quantities define directly the bulk and shear viscosities if the NavierStokes limit is considered. Straightforward manipulations lead to the following expression for the bulk viscosity coefficient [1]

$$
\zeta=\frac{g_{0} \gamma_{\mathrm{G}}^{5}}{3 \pi^{2}} \frac{\tau_{\mathrm{rel}}}{T} \int_{0}^{\infty} \mathrm{d} y\left[c_{s}^{2}-\frac{1}{3} \frac{y^{4}-1}{y^{4}+1}\right] f_{\mathrm{GZ}}\left(1+f_{\mathrm{GZ}}\right)
$$

where $f_{\mathrm{GZ}}=\left\{\exp \left[\gamma_{\mathrm{G}} \sqrt{y^{2}+y^{-2}} / T\right]-1\right\}^{-1}$. In the similar way we obtain the shear viscosity coefficient $[1,2]$

$$
\eta=\frac{g_{0} \gamma_{\mathrm{G}}^{5}}{30 \pi^{2}} \frac{\tau_{\mathrm{rel}}}{T} \int_{0}^{\infty} \mathrm{d} y \frac{\left(y^{4}-1\right)^{2}}{y^{4}+1} f_{\mathrm{GZ}}\left(1+f_{\mathrm{GZ}}\right) .
$$




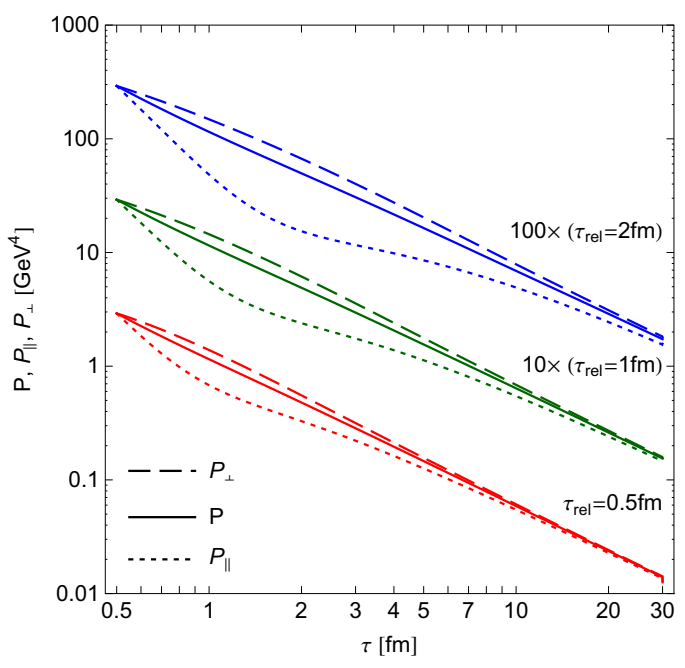

Figure 1. Time dependence of pressure for three different values of the relaxation times. The initial condition corresponds to the equilibrium state with the initial temperature $T_{0}=T\left(\tau_{0}\right)=600 \mathrm{MeV}$. The initial time is $\tau_{0}=0.5 \mathrm{fm} / \mathrm{c}$.

\section{Numerical results}

Let us now turn back to the discussion of the exact solutions of the kinetic equation. Knowing the time dependence of the effective temperature $T$ we find the longitudinal and transverse pressures from the equations

$$
\begin{aligned}
& P_{\|}=\int \mathrm{d} K \frac{w^{2}}{\tau^{2} E\left(\tau, w, k_{\perp}\right)}\left[1-\frac{\gamma_{\mathrm{G}}^{4}}{\left(w^{2} / \tau^{2}+k_{\perp}^{2}\right)^{2}}\right] f, \\
& P_{\perp}=\int \mathrm{d} K \frac{k_{\perp}^{2}}{2 E\left(\tau, w, k_{\perp}\right)}\left[1-\frac{\gamma_{\mathrm{G}}^{4}}{\left(w^{2} / \tau^{2}+k_{\perp}^{2}\right)^{2}}\right] f,
\end{aligned}
$$

and the total pressure is obtained as $P=\frac{1}{3}\left(P_{\|}+2 P_{\perp}\right)$. We note that the parallel pressure acts in the direction of the beam axis. The transverse pressure acts in the transverse direction to the beam and is the same for all such directions. The numerical results for the time evolution of pressures is shown in Fig. 1. Knowing $P_{\|}$and $P_{\perp}$ we find the shear and bulk viscous pressures (in the general case the shear tensor $\pi^{\mu \nu}$ has 5 independent components, they are reduced to one independent variable $\pi$ for $(0+1) \mathrm{D}$ systems $)$

$$
\begin{aligned}
\pi & =\frac{2}{3}\left(P_{\perp}-P_{\|}\right), \\
\Pi & =P-P_{\mathrm{GZ}} .
\end{aligned}
$$

The last two equations allow us to define the effective shear and bulk viscosities

$$
\begin{aligned}
& \pi=\frac{4}{3} \frac{\eta_{\mathrm{eff}}}{\tau}, \\
& \Pi=-\frac{\zeta_{\mathrm{eff}}}{\tau} .
\end{aligned}
$$




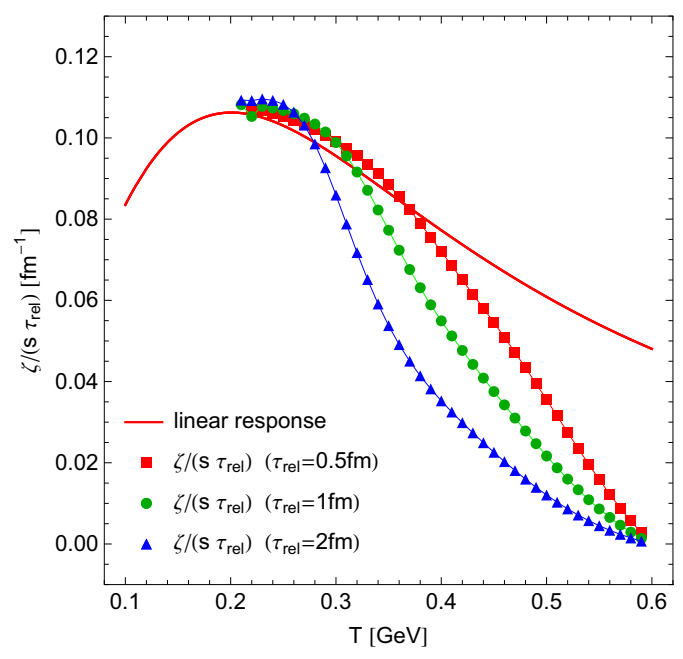

Figure 2. Temperature dependence of the bulk viscosity scaled by the relaxation time and entropy density. The triangles, circles and squares show the effective bulk viscosity for three different values of the relaxation time (defined in the figure), whereas the solid line shows the result of the linear-response formula.

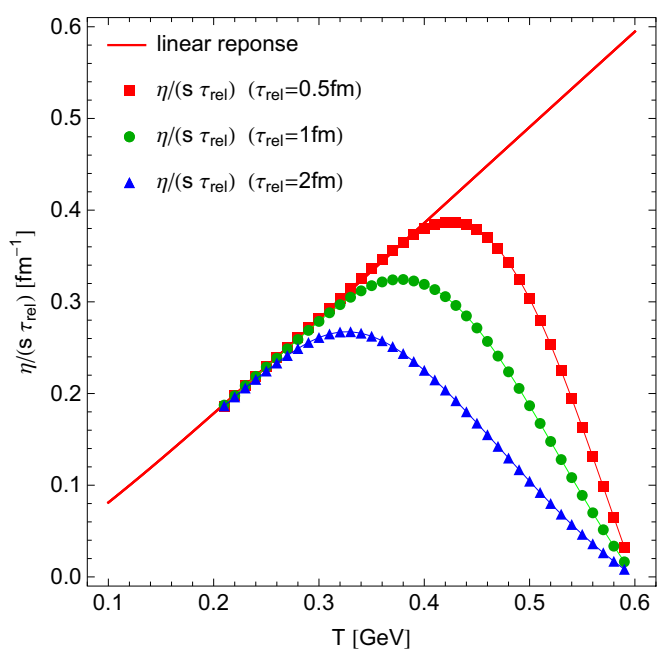

Figure 3. The same as Fig. 2 but for the shear viscosity.

The effective coefficients should agree with the standard coefficients when the system is close to equilibrium. This is demonstrated in Figs. 2 and 3. 


\section{Conclusions}

The Gribov-Zwanziger quantisation of the Yang-Mills theory leads to the dispersion relation that can be successfully used in the studies of gluon thermodynamics and kinetic theory. In this contribution we have presented the results for the bulk and shear viscosities of the Gribov-Zwanziger plasma. These results may be useful for future phenomenological applications for ultrarelativistic heavy-ion collisions.

Acknowledgements: I thank my collaborators, Radoslaw Ryblewski, Nan Su, and Konrad Tywoniuk for very fruitful collaboration. This research has been supported in part by Polish National Science Center grants No. DEC-2012/05/B/ST2/02528 and No. DEC-2012/06/A/ST2/00390.

\section{References}

[1] W. Florkowski, R. Ryblewski, N. Su, K. Tywoniuk (2015), 1504.03176

[2] W. Florkowski, R. Ryblewski, N. Su, K. Tywoniuk (2015), 1509.01242

[3] V.N. Gribov, Nucl. Phys. B139, 1 (1978)

[4] D. Zwanziger, Nucl. Phys. B323, 513 (1989)

[5] R.P. Feynman, Nucl. Phys. B188, 479 (1981)

[6] Y.L. Dokshitzer, D.E. Kharzeev, Ann. Rev. Nucl. Part. Sci. 54, 487 (2004), hep-ph/0404216

[7] N. Su, K. Tywoniuk, Phys. Rev. Lett. 114, 161601 (2015), 1409. 3203

[8] D. Zwanziger, Phys. Rev. Lett. 94, 182301 (2005), hep-ph/0407103

[9] K. Fukushima, N. Su, Phys.Rev. D88, 076008 (2013), 1304.8004

[10] S. Borsanyi, G. Endrodi, Z. Fodor, S.D. Katz, K.K. Szabo, JHEP 07, 056 (2012), 1204.6184

[11] P.B. Arnold, C. Dogan, G.D. Moore, Phys. Rev. D74, 085021 (2006), hep-ph/0608012

[12] H.B. Meyer, Phys. Rev. Lett. 100, 162001 (2008), 0710. 3717

[13] D. Kharzeev, K. Tuchin, JHEP 09, 093 (2008), 0705 . 4280

[14] G. Torrieri, I. Mishustin, Phys. Rev. C78, 021901 (2008), 0805 . 0442

[15] G.D. Moore, O. Saremi, JHEP 09, 015 (2008), 0805 . 4201

[16] J.D. Bjorken, Phys. Rev. D27, 140 (1983)

[17] A. Bialas, W. Czyz, A. Dyrek, W. Florkowski, Nucl. Phys. B296, 611 (1988)

[18] P.L. Bhatnagar, E.P. Gross, M. Krook, Phys. Rev. 94, 511 (1954)

[19] G. Baym, Phys. Lett. B138, 18 (1984)

[20] G. Baym, Nucl. Phys. A418, 525C (1984)

[21] W. Florkowski, R. Ryblewski, M. Strickland, Nucl.Phys. A916, 249 (2013), 1304.0665

[22] W. Florkowski, R. Ryblewski, M. Strickland, Phys.Rev. C88, 024903 (2013), 1305. 7234

[23] W. Florkowski, E. Maksymiuk, R. Ryblewski, M. Strickland, Phys. Rev. C89, 054908 (2014), 1402.7348 